\title{
Dynamic Measurements on a Star Tracker Prototype of AMS Using Fiber Optic Sensors
}

\author{
M.A. Caponero ${ }^{1}$, D. Colonna ${ }^{1}$, A. Cusano ${ }^{2}$, C. Gargiulo ${ }^{3}$, A. Paolozzi ${ }^{4}$, I. Peroni ${ }^{4}$ \\ ${ }^{1}$ ENEA C.R. Frascati, Via Enrico Fermi 45, 00044 Frascati (RM), e-mail: \\ caponero@frascati.enea.it, danilo.colonna@ frascati.enea.it \\ ${ }^{2}$ University of Sannio, Dipartimento di Ingegneria, Divisione Optoelettronica, Corso Garibaldi 107, \\ 82100 Benevento, email: a.cusano@unisannio.it \\ ${ }^{3}$ INFN Sezione di Roma 1, P.le Aldo Moro 5, 00185 Roma, e-mail: \\ corrado.gargiulo@roma1.infn.it \\ ${ }^{4}$ Dipartimento di Ing. Aerospaziale e Astronautica, Università La Sapienza, Via Eudossiana 18, \\ 00184 Roma, e’mail: antonio.paolozzi@uniroma1.it, isidoro.peroni@uniroma1.it
}

\begin{abstract}
An aluminum prototype of the Amica Star Tracker Support (ASTS) of the AMS_02 (Alpha Magnetic Spectrometer) experiment has been instrumented with two Fiber Bragg Gratings (FBGs). AMS_02 is a 7 tons particle physics experiment that is planned to run on the International Space Station for 3 years starting from October 2005. The star tracker, as all the sub-detectors in AMS, has to satisfy the structural verification plan requirements. In the qualification process of the star tracker FBG sensors may be used to measure static and dynamic strain in the structure during the tests. FBGs are basically optical strain gages that offer several advantages over the conventional ones such as immunity from electromagnetic interferences, small dimensions, low specific weight and the possibility of multiplexing many of them along the same fiber. In this work the use of FBGs in the acquisition of strains during a dynamic test on the ASTS semplified prototype is reported. The excitation has been provided by an instrumented impact hammer while the response has been given by one FBG and one accelerometer nominally in the same location. All the time histories have been recorded and transformed in the frequency domain to retrieve the Frequency Response Functions (FRFs) and the Strain Frequency Response Functions (SFRFs), both providing immediately the resonant frequencies of the structure. The contemporary use of the two sensors with many excitation points allow to retrieve accurate displacement and strain mode shapes with the relative resonant frequencies. As future work we plan to use a higher number of sensors in different locations and orientations.
\end{abstract}

\section{INTRODUCTION}

AMS_02 is a particle detector in space and consequently each subsystem has to be qualified for flight [1] (AMS_01, a simplified version of AMS_02, flew on board the Space Shuttle Discovery during flight STS-91 in June 1998). The subsystem considered in this paper is the AMICA (Astro Mapper for Instrument Check of Attitude) Star Tracker Camera (ASTC). The analysis is addressed to the mechanical support structure, i.e., the Amica Star Tracker Support (ASTS). ASTC is developed by the Center for Advanced research in Space Optics (CARSO) in Trieste Italy [2]. The design of a devoted mechanical support ASTS is responsibility of INFN of Rome [3]. A first simplified aluminum prototype of the ASTS has been manufactured to verify some mechanical aspects such as the clamping on the main structure of AMS and the cabling from the fiber optic sensors to the interrogation system. Optical fibers can be used in the qualification process since they 
are capable to measure static and dynamic strains when embedded or stuck on a structural component. If the fiber is used without sensors, the typical way to make a measurement is to insert the component into an interferometer [4-6]. The sensorless optical fibers have the advantage of being inexpensive but interferometers are difficult to be used outside a laboratory and provide only the total length variation of the portion of the fiber attached on the structure. Consequently the corresponding Fast Fourier Transform (FFT) of the responses can be used to determine the natural frequencies of the structure but not the mode shapes. Therefore interferometric techniques are not appropriate to perform a modal analysis. To overcome this limitation point measurements are required. To this end Fiber Bragg Gratings (FBGs), that are basically optical strain gages, can be used as an alternative [7-10] to conventional sensors such as accelerometers, or to other techniques such as laser vibrometry, holographic interferometry and Electronic Speckle Pattern Interferometry (ESPI) [11-19]. Of course in the case of FBGs one obtains, in the frequency domain, the Strain Frequency Response Functions (SFRFs) as a ratio of the Fast Fourier Transform (FFT) of the responses with the FFT of the excitations (the procedure is more complex if one uses random excitation) [20]. By following the same procedure used with conventional FRFs, one retrieves strain modes that are defined as the distribution of strains corresponding to a given deformation mode. The advantage of having a structure instrumented with FBGs is the possibility of integrating (attaching) them inside (on the surface of) the material, with minimal harness. Such a system can therefore operate before and during the operating life of the component. Thinking at our application these sensors are useful in the qualification phase. In fact as every space component the ASTC will be subjected to the launch environment and therefore one can use the FBGs to check if its modal characteristics are compatible with such an environment. Furthermore having a good modal model and the corresponding strain modal model it is possible to build a transformation matrix that allow to transform any strain distribution into a deformed shape or vice-versa [21,23].

\section{AMS EXPERIMENT}

AMS_02 is a particle detector for the International Space Station (ISS). It is located on the main truss of the ISS as shown in Fig. 1.

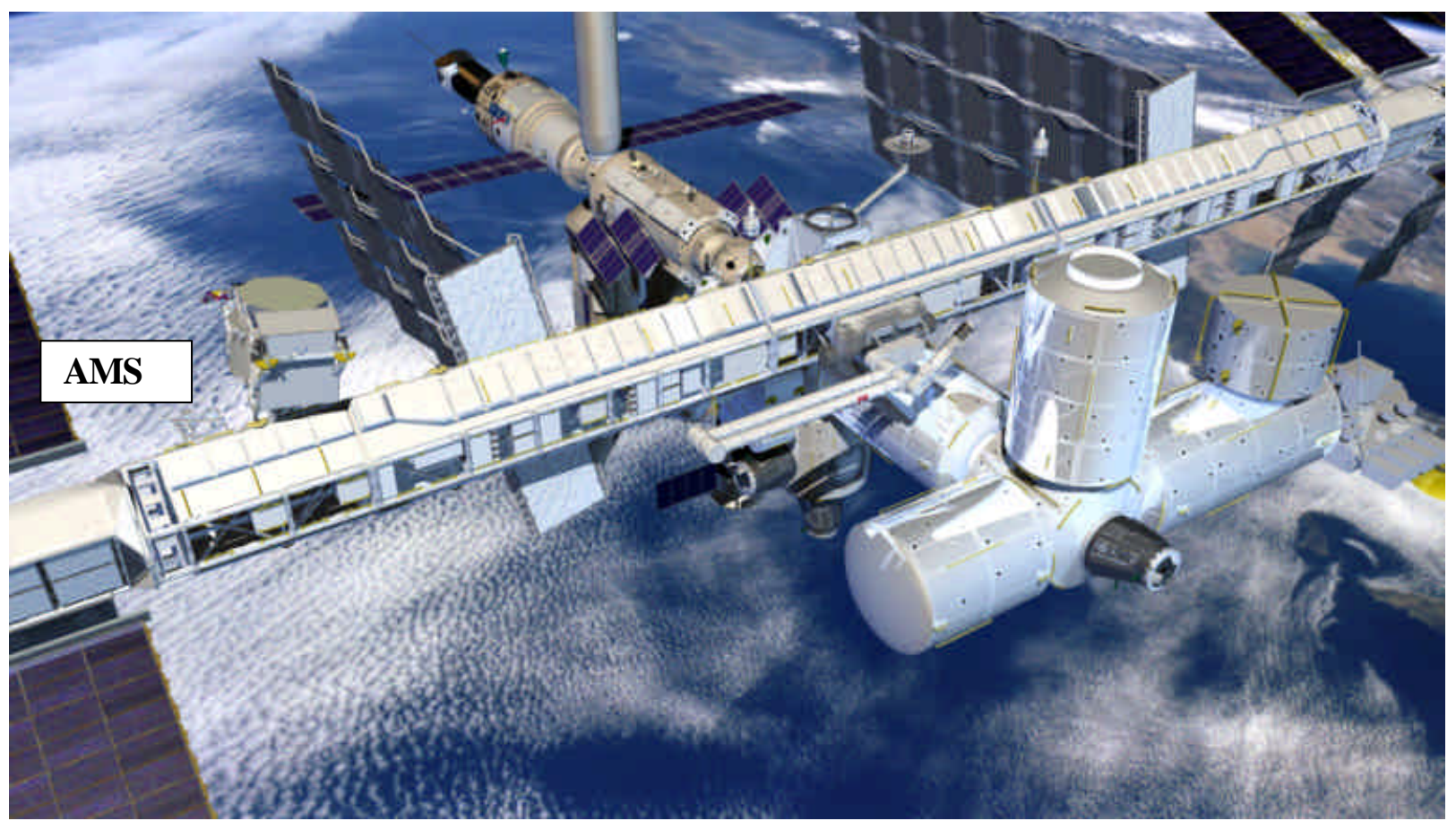

Fig. 1. View of the AMS experiment on the International Space Station. 
It is constituted of several subdetectors: Transition Radiation Detector (TRD); Time of Flight detector (TOF); Anti-Coincidence Counter (ACC); Silicon Tracker (ST); Ring Imaging Cherenkov counter (RICH); Electromagnetic Calorimeter (E-Cal) and a cryogenic super conducting magnet at its core (Fig. 2). A particle detector, whether it is in space or on a large ground based accelerator, is characterized by high stiffness and very low mass. That is important to reduce perturbations on the particles travelling through it. Furthermore when a detector is sent to space high specific stiffness is a requirement that applies also to support structures that are not strictly devoted to particle tracking. While in the accelerators the observed elementary particles are generated artificially, for instance through the collision of protons and antiprotons, in AMS_02 high-energy particles coming from outer space as cosmic rays are detected. Their origin is either in supernova explosions inside galaxies or in hypothetical quark stars or dark matter annihilation or even by galaxies made of antimatter. Antimatter can be generated artificially through collisions of accelerated particles or can be observed in the decay of some radioactive elements. In general whenever energy is transformed into mass it always generates an equal amount of matter and anti-matter. The same thing should have happened at the Big Bang. But up to now no evidence of antimatter in the universe has been found. So either the Big Bang somehow did not make (or destroyed) the expected antimatter, or made antimatter in a different region of the universe far away from our galaxy or our cluster of galaxies. While the first hypothesis is sort of theoretical (it would require both violation of baryon number and $\mathrm{CP}$ violation) and can be verified with experiments such as BABAR at Slac, BELLE at KEK, BTeV [24] at Fermilab and LHCb at Cern, the second one can be verified with AMS_02. The meaning of AMS is Alpha Magnetic Spectrometer because it is supposed to detect complex nuclei like anti-helium, i.e., anti-alpha particles, or anti-carbon. In fact while anti-proton or anti-electron can be produced by high energy collision of particle made of matter, anti-nuclei are almost never created in those collisions and therefore they will be a strong evidence of the existence of anti-star because they will produce huge quantities of anti-helium by nuclear fusion of anti-hydrogen.

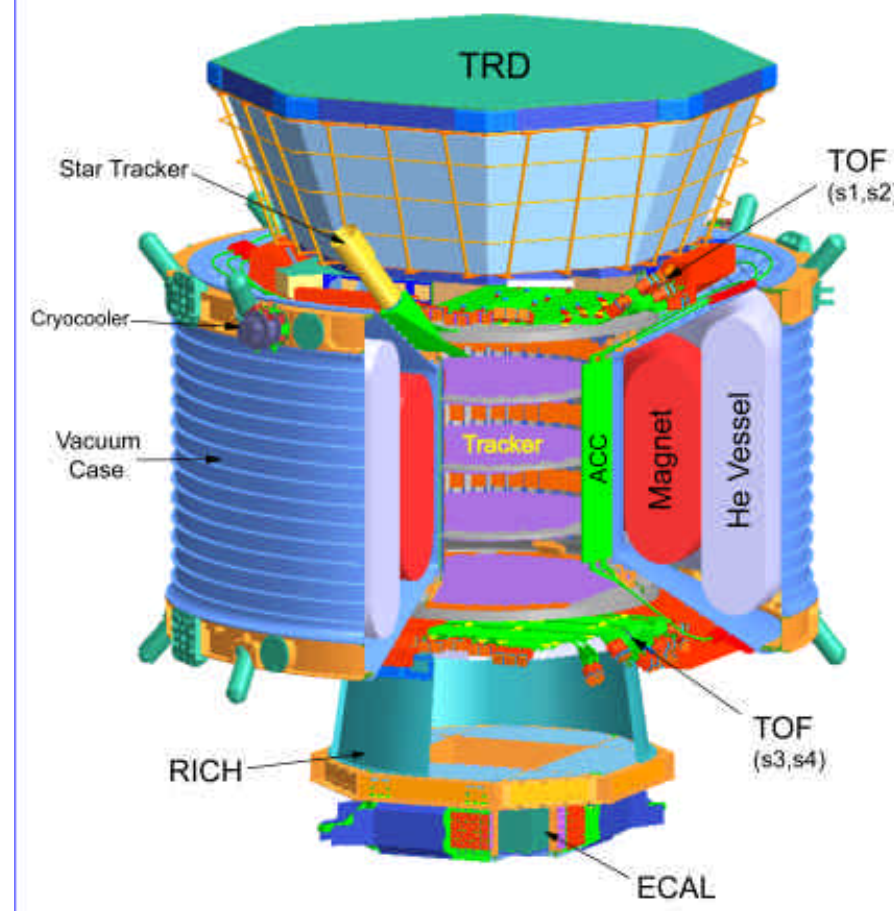

Fig. 2a. Artist view of AMS_02.

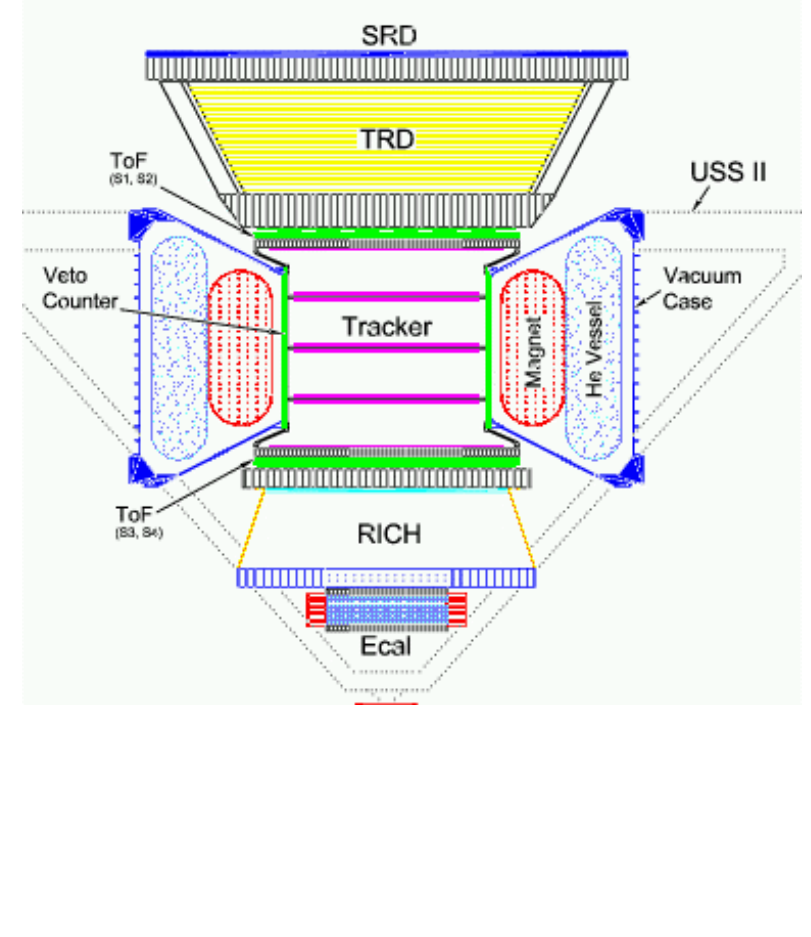

Fig. 2b. Cross section of Fig. 2a. 
The other important topic is to understand the nature of the cold dark matter that is necessary to explain the gravitational motion within galaxies and cluster of galaxies as well as other astrophysical observations. An hypothesis suggested by theorist is that dark matter is made by "neutralinos" that are massive, stable, neutral particles predicted by SUperSYmmetry (SUSY). If neutralinos exist, AMS should observe extra positrons (or if you prefer anti-electrons) at high energy, because these are produced by the collision of neutralinos. AMS will also search for strangelets, i.e., matter made of strange quarks. This matter is very dense and with lower charge and is stable at any size so that a nucleous can weight $10-100$ times a regular one and carry $1 / 2$ or $1 / 3$ of the expected charge.

AMS will be also a gamma ray observatory. Gamma rays are observed exploiting the transformation of energy into mass.

\section{STAR TRACKER}

The Star Tracker (ASTC) is an imaging system that provides inertial reference for a spacecraft. The ASTC Two different ASTC will be mounted on AMS_02 as shown in Fig. 3. They both attach to the upper portion of the AMS_02 "Silicon Tracker" (ST) sub-detector. The ST is capable to determine with high accuracy the particle trajectory, but in order to determine the source location in the sky one needs to know the orientation of AMS with respect to a given reference frame (usually an inertial frame) and this is the function of the ASTC. The ASTC components are basically the $\mathrm{CCD}$, the lens system, the electronics and the baffle. The baffle, made of black anodised aluminum, avoids that direct light or light reflected from the space station enters the lens system. Some of the requirements established for the ASTC follow [24]: angular camera stability: 36 arcseconds (0.01deg); baffle stability: angular: $1.5^{\circ}$, translation: several millimeters (design dependent); camera operational temperature range: $-20^{\circ}$ to $40^{\circ} \mathrm{C}$; camera survival temperatures: $\quad-40^{\circ} \mathrm{C}$ to $40^{\circ} \mathrm{C}$; power: 4W each. A star-matching algorithm is also included in the ASTC. Some aspects of the ASTC are still to be defined such as the thermal design. Limitations on ASTC working temperature suggest the connection of the system to ST cooling circuit located only few centimeters away from the star tracker. This could permit to cool the $8 \mathrm{~W}$ power, and obtain temperature stability and possibly better structural stability.

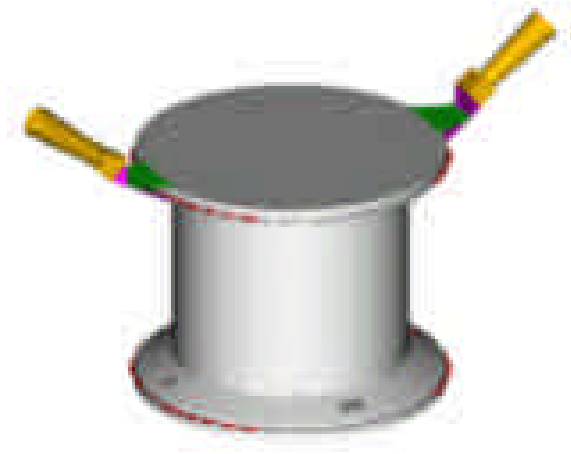

Fig. 3a. The two star trackers mounted on AMS.

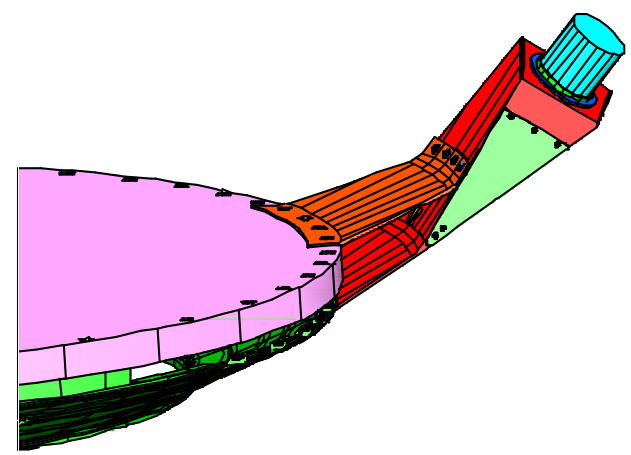

Fig 3b. Magnified view of the star tracker without buffle. 


\section{EXPERIMENTAL SET-UP}

In Fig. 4a is reported a scheme of the star tracker, while in Fig. $4 \mathrm{~b}$ is reported a photograph of the prototype. In this last figure is visible the dummy mass used to simulate the lens. The material used for the prototype was an $\mathrm{Al}$ Alloy 5083. In Fig. 5a is reported the prototype fixed on a mock up of the supporting structure. It is clearly visible the experimental grid used to perform the modal analysis. The two cables are the protecting jackets containing two optical fibers (diameter of the optical fiber without coating $125 \mu \mathrm{m}$ ). They are commonly used in the telecommunication technology for handling the fibers without breaking them. Inside the two fibers, two Fiber Bragg Gratings (FBGs) have been manufactured (a brief explanation on the manufacturing and the woking principle of an FBG is given right below). The jacket (typical diameter $900 \mu \mathrm{m}$ ) has been removed near the FBGs and the coated fiber $(250 \mu \mathrm{m}$ in diameter, barely visible in Fig. $5 \mathrm{~b}$ near the accelerometer) has been glued on the aluminium surface. The removal is obviously necessary to measure meaningful strains.

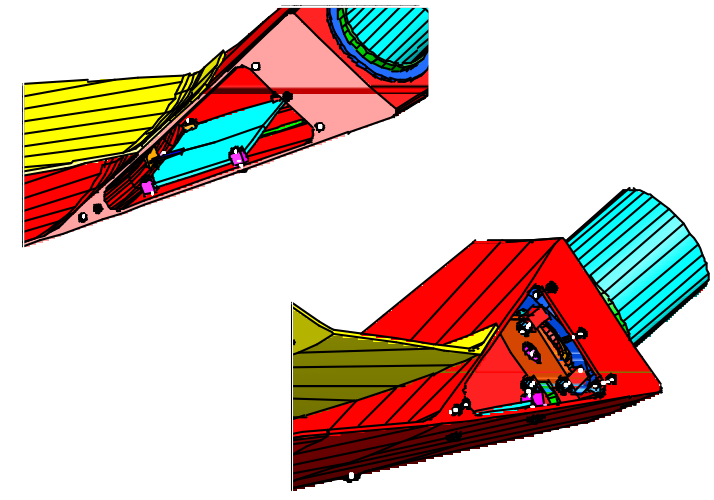

Fig. 4a. Details of the star tracker.

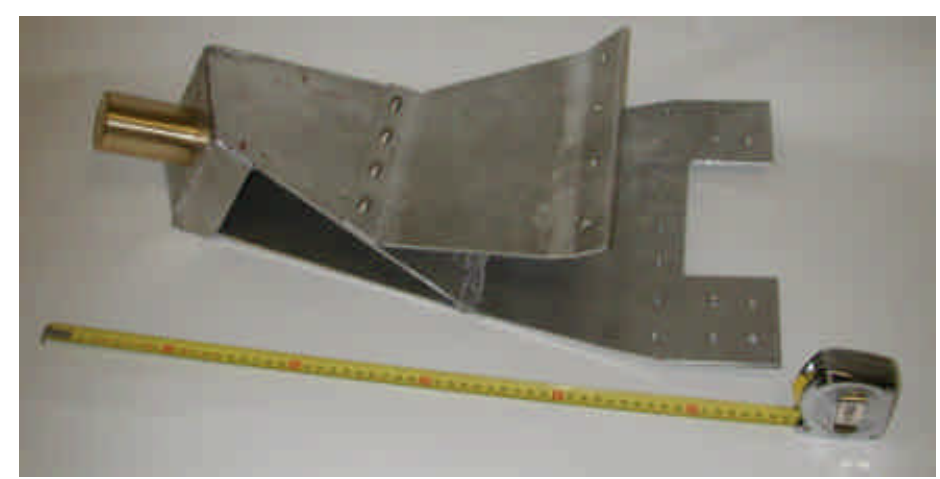

Fig. 4b. Assembled prototype of star tracker.

An FBG sensor consists of a longitudinal periodic modulation of the refractive index of the core of an optical fiber (typical core diameter for single mode fibers is less than $10 \mu \mathrm{m}$ ). Such a modulation is induced by an interference pattern of coherent ultraviolet light produced right at the core of the fiber. The usual length of the pattern is about $10 \mathrm{~mm}$. It is important to observe that the FBG is inside the fiber itself and is invisible. So that two pencil marks are required to locate it. These type of sensors are called intrinsic sensors, as opposed to the extrinsic sensors that are instead bigger and well visible. Now few words on the working principle. Wide-band infrared light propagates along the optical fiber, it is diffracted by the FBG and, as a result, narrow-band light is back-reflected along the optical fiber. The wavelength $\lambda_{\mathrm{B}}$ of the back-reflected light, called Brag wavelengh, is a function of the grating period $\Lambda$ and of the effective refractive index $n_{\text {eff }}$ of the fiber at the grating:

$$
\lambda_{\mathrm{B}}=2 \cdot n_{\text {eff }} \cdot \Lambda
$$

FBGs can be easily used not only for strain measurements but also for temperature sensing if a suitable structural or thermal bond is provided between the structure to be monitored and the piece of the optical fiber where the FBG is located. Any deformation or temperature variation of the optical fiber will affect the value of $n_{\text {eff }}$ and/or $\Lambda$, thus modifying the back-reflected wavelength $\lambda_{\mathrm{B}}$. As a sensing device, FBGs have unrivalled long term stability, multiplexing read-out capability, electromagnetic field insensitivity, hostile environment endurance and mass lightness. In this work we did not exploit the multiplexing capability of the FBG technique since we wanted to observe the 
experimental results using the simpler Single-Input-Single-Output (SISO) modal analysis technique but of course the contemporary use of more FBG sensors multiplexed along the same fiber would allow, with just one excitation to retrieve many FRFs without increasing the harness. An important component of the experimental set-up is the FBG interrogation system and the broad band infrared source. In Ref. [11] for the first time a strain mode shape was retrieved on a Carbon Fiber Reinforced Plastics beam using the responses of an FBG. However the CFRP beam was designed to have the first two mode shapes below $20 \mathrm{~Hz}$. This threshold was imposed by the acquisition rate of an FBG interrogation system built and commercialized by Micron Optics. The resonances of the ASTS were higher as it could be immediately realized from the finite element simulation. It was therefore clear the need of a high frequency interrogation system. Such a system has been developed at the university of Sannio [25,26] and relies on a low cost approach based on grating based optical filtering combined with broadband interrogation. By using chirped and strongly apodized FBG, written in optical fibers curved in the plane of the phase mask, an in fiber optical filter with transmission and reflection spectrum linearly varying with optical wavelength can be achieved. The grating based filter was designed to exhibit a transmission and reflection responses linearly varying in the range $1547-1552 \mathrm{~nm}$ (5nm linearity range) with opposite slope coefficients. A proper a-thermal package has been chosen in order to ensure a good thermal stability. The slope of the optical filter in transmission, directly calculated as the rise in percent over the run in nanometers, is of approximately of $0.16 / \mathrm{nm}$ (Reflectivity exhibits a wavelength slope of $-0.16 / \mathrm{nm}$ ). This slope is valid from about the $10 \%$ up to $90 \%$ range. When the reflected signal by the sensing grating interacts with the optical filter, wavelength shifts are converted in amplitude modulation at the output of the grating based filter. By properly choosing linearity range and slope, customized interrogation units could be designed for different requirements in terms of resolution and measuring range. Due to the use of passive optical filtering, system bandwidth is only due to the electronic circuitry involved in the detection unit actually limited to $400 \mathrm{KHz}$. In Fig. $5 \mathrm{~b}$ is reported a detail showing the accelerometer.

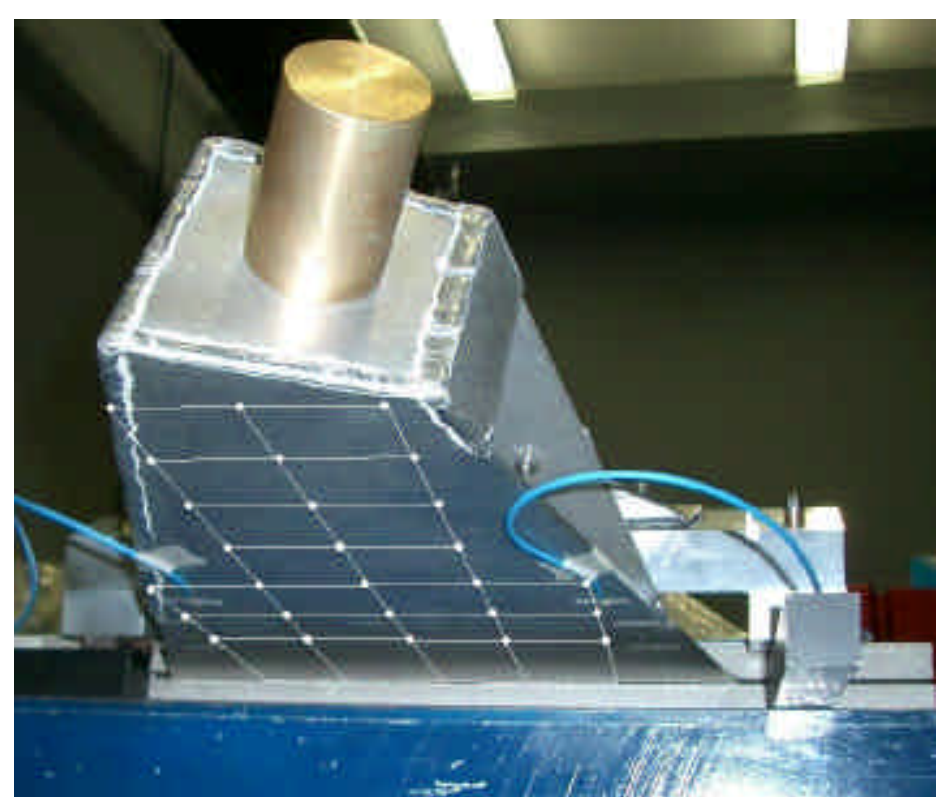

Fig. 5a. Experimental grid on star tracker prototype.

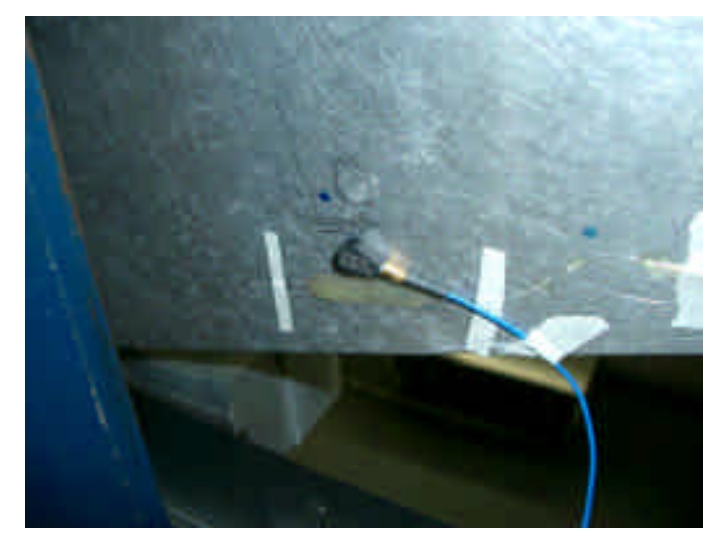

Fig. 5b. Detail showing the accelerometer. 


\section{NUMERICAL AND EXPERIMENTAL RESULTS}

A Finite Element (FE) Analysis has been developed to predict ASTS natural frequencies. The structure has been mapped meshed using thin shell linear elements, Mindlin theory based (Ideas_element type: 94-thin_shell); lens mass is modeled as lumped mass (I-deas_element type: 161lumped_mass) in its center of mass and is connected to the structure by rigid elements (I-deas elements types: 121_rigid bar and 23_rigid_element).The mechanical characteristics are as follows: ASTS structure: density $\rho=2700 \mathrm{~kg} / \mathrm{m}^{3}$, Young modulus $\mathrm{E}=70 \mathrm{GPa}$, Poisson ratio $v=0.33$, wall thickness $\mathrm{t}=4 \mathrm{~mm}$. Lens: lumped mass $=1.7 \mathrm{~kg}$ with an offset of $38 \mathrm{~mm}$. The lumped mass is visible in Fig. 6 (as a black square in the undeformed configuration) where the first and second mode shapes are reported.

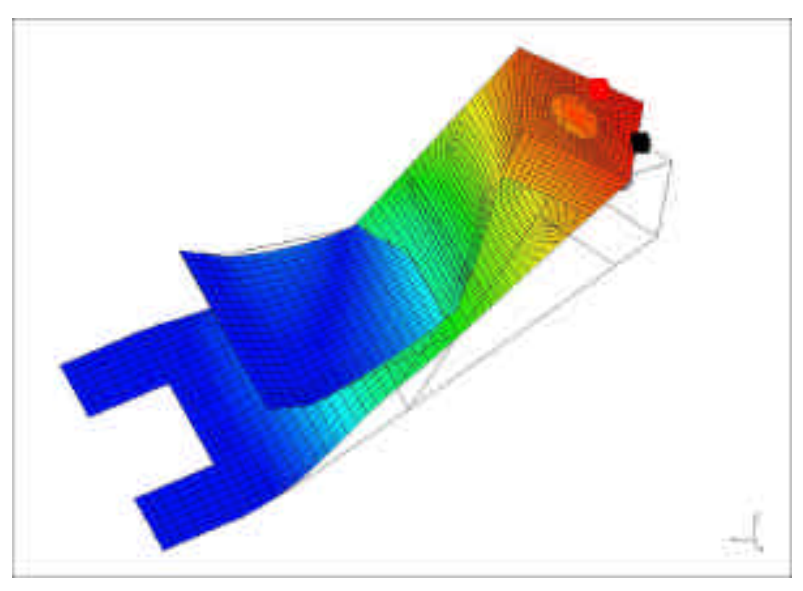

Fig. 6a. First mode shape of FEM of prototype.

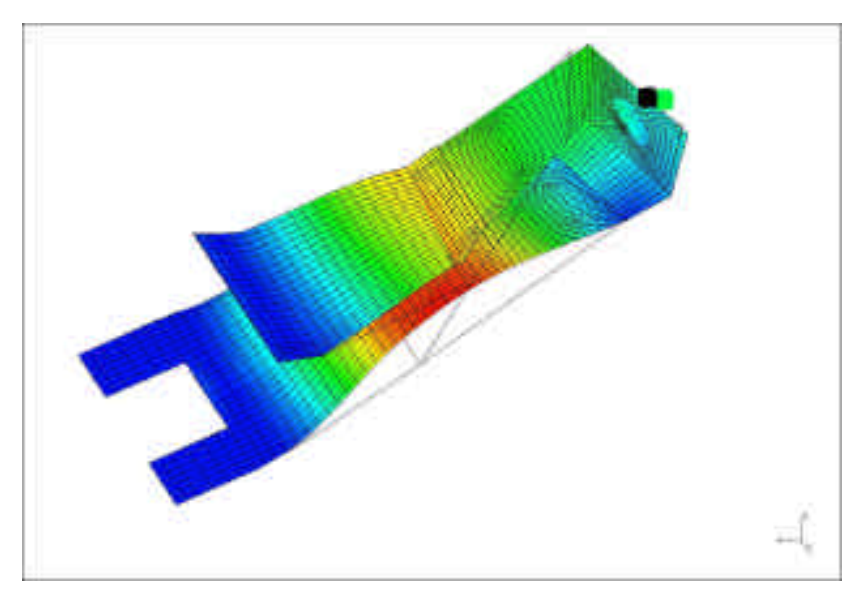

Fig. 6b. Second mode shape of FEM of prototype.

The responses of one of the two FBGs available and of the accelerometer as well as the time histories provided by the load cell of the impact hammer, have been acquired using a home made LabView program. All the time histories have been acquired without any trigger. Therefore each data set started with noise. Five acquisitions for each mode were performed. The procedure applied to retrieve the experimental displacement and strain modes is outlined below. Presently the procedure was not automated and consequently, due to the short time available, no averaging was performed and consequently only one FRF and one SFRF per excitation point have been obtained. The acquisition of the three signals were perfectly synchronized. The noise in the time interval between the acquisition and the impact was eliminated. The FFT of the time histories were calculated. The FRFs (SFRFs) were calculated as the ratio of the FFT of the response of the accelerometer (FFT of the response of the FBG) with the FFT of the excitation. Then by applying the classical modal analysis techniques, from the FRFs (SFRFs) the mode shapes (strain mode) have been retrieved. To have a rough estimation of the mode shapes one can look at the FRF (SFRF) directly without performing a fitting and use the peaks of the imaginary parts to obtain a mode shape (strain mode). The displacements (strains) have been obtained at the frequencies of 38.33 and $123.78 \mathrm{~Hz}$ at the location shown in the grid reported in Fig. 5a. The mesh consisted of 27 points plus five considered clamped.

The strain modes associated with the first two displacement modes have been obtained from he responses of the FBG (located near the accelerometer) directed in the y direction (i.e. perpendicular at the constrained edge and laying in the mesh plane). Those values represent the strains at the first two natural frequencies. While the responses of the accelerometer are proportional to the modal displacement or better to their second time derivative, the responses of the FBG at the same frequency are proportional to the strains of the associated modal displacement. Such strains are 
proportional, for modes that can be essentially considered flexural modes, to the curvature along the $\mathrm{y}$ axis and to the distance from the neutral plane. In other words they represent the second partial derivative of the vertical displacement with respect to the spatial coordinate.

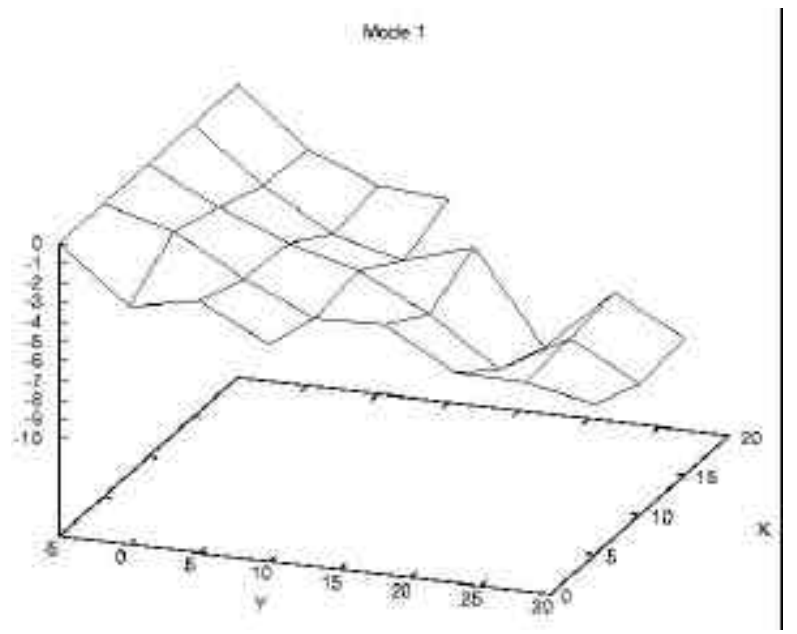

Fig. 7a. First experimental displacement mode.

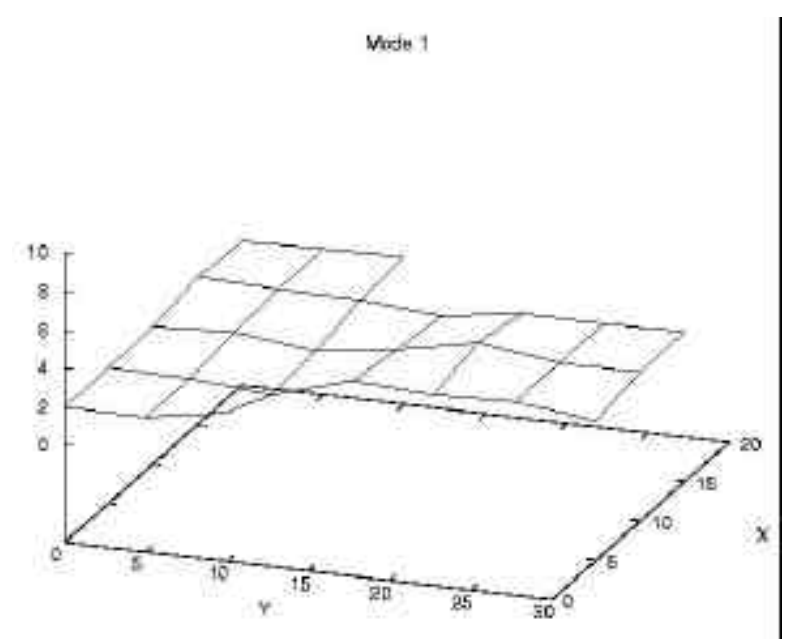

Fig. 8a. First experimental strain mode.

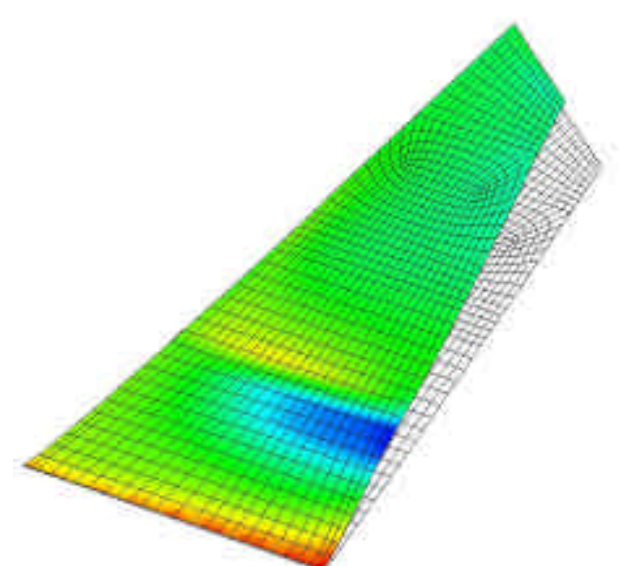

Fig. 9a. First FE displacement mode.

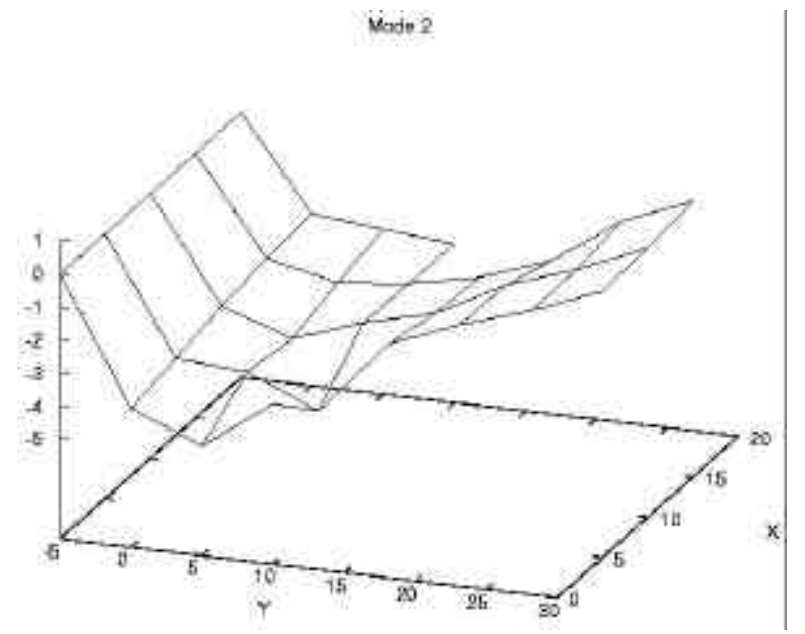

Fig. 7b. Second experimental displacement mode.

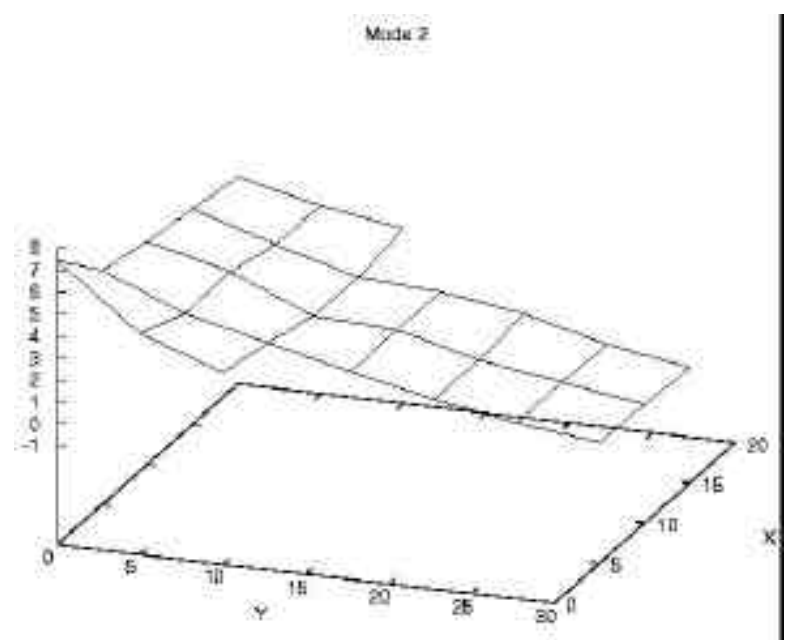

Fig. 8b. Second experimental strain mode.
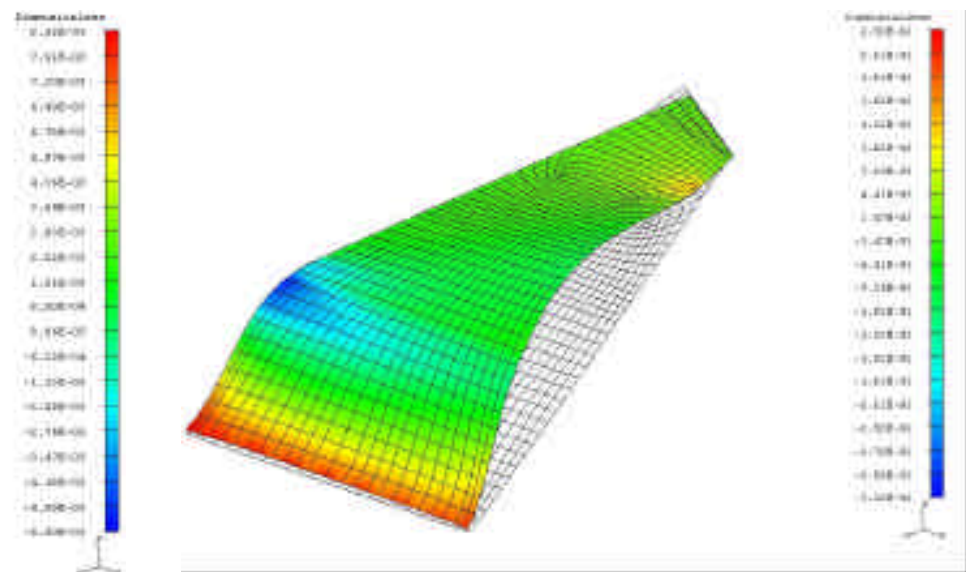

Fig. 9b. Second FE displacement mode. 
This circumstance is approximately verified in our results as can be seen in Figs 7 and 8. The strain distribution for the first mode shape is approximately constant (Fig. 8a) as one can expect from a deformed shape with approximately constant curvature (Fig. 7a). On the other hand the second mode shape (Fig. 7b) shows a variable curvature that reduces towards the tip until it becomes negative. That behavior can be clearly seen in Fig. 8b. A closer view to Figs. 7 and 8 shows that the experimental grid used in Fig. 7 has four more points. Those points, as mentioned earlier, are relevant to the clamped end and cannot be reported in Fig. 8 because at the clamped end the values of the strains cannot be guessed. Finally in Fig. 9 is reported the bottom part of Fig. 6. The grayscale represent the strain distribution as calculated by the FE method. A further quick test has been performed using the four channel NI4551 (Dynamic Signal Analyzer) card with real time FFT on-board processing capability. This test could be performed only with the accelerometer because the optical signal from the FBG sensor, even if transformed to a digital electrical signal, was not directly compatible with the National Instrument board. In Fig. 10 are plotted the imaginary parts corresponding to the first and second resonance of the FRFs acquired along the central line of the grid reported in Fig. 5a.

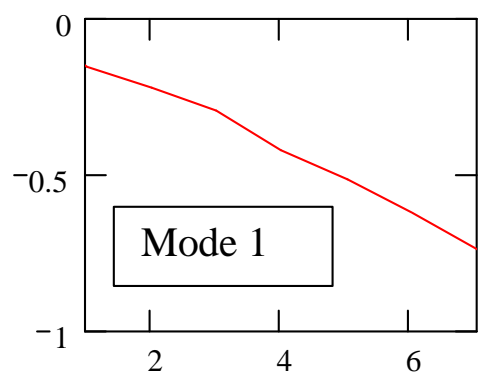

Fig. 10a. Experimental mode shape.

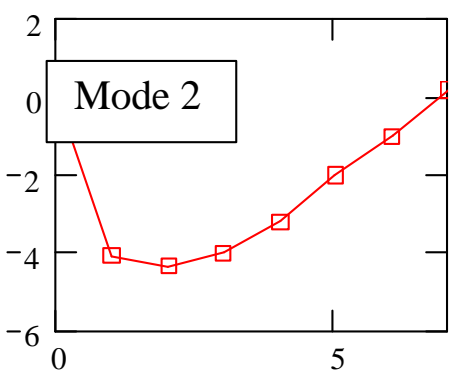

Fig. 10b. Experimental mode shape.

Finally in Tab. 1 are reported the natural frequencies obtained with the different methods. Not much effort has been devoted to improve the matching between the numerical and the experimental results because that was not the aim of the present work. The results obtained with the FBG and the accelerometer are in very good agreement if one excludes the fourth mode that shows a difference of about $5 \%$.

Table 1. Comparison of natural frequencies

\begin{tabular}{|l|l|l|l|}
\hline Mode number & Numerical & Accelerometer & FBG \\
\hline 1 & 41.74 & 38.33 & 38.33 \\
\hline 2 & 120.14 & 123.78 & 123.78 \\
\hline 3 & 190.69 & 172.12 & 171.39 \\
\hline 4 & 395.56 & 349.85 & 333.25 \\
\hline
\end{tabular}

\section{CONCLUSIONS}

In this work a numerical and an experimental modal analysis has been performed on a star tracker prototype of the AMS_02 experiment. The dynamic responses have been obtained from an accelerometer and a fiber optic sensor (specifically an FBG) glued on the surface of one of the star tracker panels. The numerical test has shown the need of a high frequency fiber optic interrogation system while the experimental results obtained with the two different sensors have shown excellent 
agreement for the first three natural frequencies and an acceptable agreement for the fourth one. The mode shapes obtained by the accelerometer and the strain mode shape obtained by the FBG responses are compatible. As a future work, an improvement of the results can be obtained by implementing a trigger for the acquisition, an averaging and a curve fitting to the FRFs and the SFRFs. The measurement and analysis of the responses in all the external surfaces of the star tracker is planned. Also a Single-Input-Multi-Output test will be performed exploiting the multiplexing of the FBGs on a single fiber. Finally an automatic procedure for data acquisition and analysis will be prepared.

\section{REFERENCES}

1. Martin T.D., "Alpha Magnetic Spectometer_02 Structural Verification Plan for the Space Transportation System and International Space Station", JSC-28792 Rev.B, NASA Space and Life Sciences Directorate Flight Project Division, December 2001-RevB

2. Trampus P., "Amica Star Tracker", AMS Technical Interchange Meeting, Johnson Space Center, October 28-31 Houston Texas.

3. Gargiulo C., "AMS Star Tracker Support Structure Design", AMS Technical Interchange Meeting, Kennedy Space Center, Jan 13-17, 2003

4. R.Becker. "Star Tracker Mechanical Requirements", AMS Technical Interchange Meeting, Johnson Space Center, Houston, Texas, October 28-31, 2002

5. Paolozzi A., Caponero M.A., Cassese F., Leonardi M., "Use of Embedded Optical fibers for Structural Analysis", XVII International Modal Analysis Conference (IMAC), Orlando, FL, Feb. 1999, Vol. 1, pp. 699-704.

6. Caponero M.A., Felli F., Paolozzi A., "Vibration Tests on Metal Alloys with Embedded Optical Fibers", SPIE's Symposium on smart Materials and MEMS (Smart Material), Melbourne, Australia, Dec.13-15, 2000, vol. 4234, pp. 152-159.

7. Paolozzi A., Felli F., "Broad Band Tests on Metallic Specimens by embedded Optical Fibers", XVI AIDAA, Torino, Sept. 2001, pp. 1-10, paper no. 103.

8. Caponero M.A., Felli F., Paolozzi A., "Strain Measurements with FBGs Embedded into Cast Metal Alloys", 7th Japan Int. SAMPE Symp. and Exhibition (JISSE 7), Tokyo Nov. 2001, pp. 661-664.

9. Paolozzi A., "Recent Researches on Fibre Optic Smart Structures in Italy and Future Trends in Europe", Key note at the 7th Japan Int. SAMPE Symp. and Exhibition (JISSE 7), Tokyo, Nov. 2001, pp. 35-42.

10. Paolozzi, A., Caponero, M.A., Felli, F., Colonna, D., "Vibration tests on a composite cantilever beam by fibre Bragg Gratings", Proc. of the Intern. Conf. on Structural Dynamics Modelling, Madeira, Portugal, June, 2002.

11. Paolozzi A., Caponero M.A., Sarasso M., Colonna D., "Static and Dynamic Measurements on an Aerospace Composite Beam by Embedded Fiber Optic Sensors", JSME/ASME International Conference on Materials and Processing 2002 (M\&P2002), Hawaii, October 15-18, 2002, pp. 384-389.

12. Caponero M.A., Paolozzi A., Peroni I. "Use of Speckle Interferometry and Modal Assurance Criterion for Identification of Component Modes", Journal of Optics and Lasers in Engineering, vol. 37, 2002, pp. 355-367.

13. Caponero MA., Paolozzi A., Pasqua P., Peroni I., "Use of Holographic Interferometry and Electronic Speckle Pattern Interferometry for Measurements of Dynamic Displacements", Mechanical System and Signal Processing, vol. 14, 2000, pp. 49-62 ISSN: 0888-3270.

14. Caponero M.A, Felli F., Paladini A., Paolozzi A., "Use of ESPI for absolute high resolution calibration of FBG sensors", $15^{\text {th }}$ International Conference on Optical Fiber Sensors at the Hilton Portland, Portland, Oregon, USA, April 2002. 
15. Caponero M.A., Paolozzi A., Ivagnes M., Lecci U., "Mechanical Characterization of Aerospace Sandwich Panels by Speckle Interferometry", 19th International Modal Analysis Conference (IMAC), Feb. 2001, pp. 1311-1317.

16. Agneni A., Caponero M.A., Paolozzi A., "Image processing for Fringe Unwrapping in Speckle Interferometry", XVIII International Modal Analysis Conference (IMAC), S. Antonio, TX, Feb. 2000, vol. 2, pp. 1479-1484.

17. Caponero M.A., Palmerini G.B., Paolozzi A., Peroni I., "Influence of the Constraints on the Modal Parameters of a Microsatellite", XV Congresso Nazionale AIDAA, Torino, Italy, Ott, 1999, pp. 1245-1256.

18. Caponero M.A., Paolozzi A., Pasqua P., Peroni I., "Modal Pattern Detection of Aerospace Sandwich Structures by Speckle Interferometry", 16th International Modal Analysis Conference (IMAC), Santa Barbara, CA, Feb 1998, vol. 2, pp. 1257-1263.

19. Caponero M.A., Paolozzi A., Peroni I., "Modal Tests on the Full-scale Model of a Microsatellite", 15th International Modal Analysis Conference (IMAC), Orlando, Florida, Feb. 1997. (pp. 470-475).

20. Caponero M.A., Paolozzi A., Peroni I., Rizzo R., "Mode Shape Experimental Holographic Technique for Spacecraft Sandwich Panels", 14th International Modal Analysis Conference (IMAC). Dearborn, Michigan, Febbraio 1996, vol. 2, pp. 1539-1545.

21. Bernasconi, O., Ewins, D.J., "Application of Strain Modal Testing to Real Structures", Sixth International Modal Analysis Conference, 1988, pp. 1453-1464

22. Borget P.B., Haugse E., Gehrki, R.E., "Structural Shape Identification from Experimental Strains Using Modal Transformation Technique", AIAA 2003

23. Kirby, G.C., Lim, T.W., Weber, R., Bosse, A.B., Povich, C., Fisher, S. "Strain-based Shape Estimation Algorithms for a Cantilever Beam", Proceedings of the linternational Society for Optical Engineering Conference, March 1997

24. Benussi L., Bertani M., Bianco S., Caponero M.A., Fabbri F.L., Felli F., Giardoni M., La Monaca A., Pace E., Pallotta M., Paolozzi A., "Use of Fiber Bragg Grating Sensor for Position Monitoring in High Energy Physics Experiment BTeV", IEEE Sensor 2002, Kissimmee, FL, June 2002, (pp. 874-879).

25. Cusano A., Breglio G., Cutolo A., Calabrò A., Giordano M., Nicolais L., "All Fiber Bragg Grating Sensing System for Static and Dynamic Strain Measurements", Proc. of the Third International conference on Structural Health Monitoring, Stanford University, September 10-12, 2001, pp. 1158-1164.

26. Cusano A., Persiano G.V., Breglio G., Nasser J., Giordano M. "Dynamic Strain Measurements by Fibre Bragg Grating Sensor", Eurosensors XVI Conference, Prague, September 2002 pp. 345-349. 